\title{
Quadrupole mass filter with fuzzy initial conditions
}

Sarkhosh Seddighi Chaharborj ${ }^{1}, 2,3^{*}$, Fudziah Ismail ${ }^{1}$, Fakhreddin Abedi ${ }^{1}$ and Mahendran Shitan ${ }^{4}$

"Correspondence:

sseddighi2007@yahoo.com

${ }^{1}$ Department of Mathematics, Faculty of Science, Universiti Putra

Malaysia, Serdang, 43400 UPM,

Malaysia

2Plasma Physics and Nuclear Fusion Research School, Nuclear Science and Technology Research Institute (NSTRI), P.O. Box 14395-836, Tehran, Iran

Full list of author information is available at the end of the article

\begin{abstract}
The employment of the fuzzy method to solve differential equations has been well studied. In this article, Mathieu differential equations of the quadrupole mass filter (QMF) have been solved by using the fuzzy method. This method has not been yet investigated in the QMF with fuzzy initial conditions. We survey the physical properties of the confined ion. The results of numerical simulations are presented and discussed.
\end{abstract}

Keywords: quadrupole mass filter; Mathieu differential equations; ordinary differential equation; fuzzy initial conditions; ion trajectory

\section{Quadrupole mass filter with hyperbolic rods}

The quadrupole mass filter (QMF) principle was established by Paul and Steinwedel [1]. It consists of four parallel hyperbolic cross-section rod electrodes as shown in Figure 1. If opposite pairs of electrodes have steady potentials $\Phi_{0}$ and $-\Phi_{0}$, it follows that the potential in the space between them is

$$
\Phi(x, y)=\Phi_{0}\left(r^{2}+z^{2}\right)
$$

where $r$ and $z$ are position coordinates from centerline and

$$
\Phi_{0}=U_{\mathrm{dc}}+V_{\mathrm{ac}} \cos (\Omega t)
$$

where $U_{\mathrm{dc}}$ is direct potential, $U_{\mathrm{ac}}$ is the zero to peak amplitude of the RF voltage, and $\Omega$ is RF angular frequency [1-5]. Equation (1) satisfies the Laplace equation and boundary conditions of the system. In each of the perpendicular directions $r$ and $z$, the equation of motion for a particle of mass $m$ and charge $e$ in such a time varying field may be written

$$
\begin{aligned}
& \frac{d^{2} z}{d \zeta^{2}}+\left(a_{z}-2 q_{z} \cos (2 \zeta)\right) z=0 \\
& \frac{d^{2} r}{d \zeta^{2}}-\left(a_{r}-2 q_{r} \cos (2 \zeta)\right) r=0
\end{aligned}
$$

where $\zeta=\frac{\Omega t}{2}$, and

$$
a_{z}=-a_{r}=8 \frac{e}{m} \times \frac{U_{\mathrm{dc}}}{r_{0}^{2} \Omega^{2}} \quad \text { and } \quad q_{z}=-q_{r}=4 \frac{e}{m} \times \frac{V_{\mathrm{ac}}}{r_{0}^{2} \Omega^{2}} .
$$

2013 Seddighi Chaharborj et al: licensee Springer. This is an Open Access article distributed under the terms of the Creative Commons Attribution License (http://creativecommons.org/licenses/by/2.0), which permits unrestricted use, distribution, and reproduction in any medium, provided the original work is properly cited. 
Figure 1 Quadrupole mass filter with hyperbolic cross-section rods.

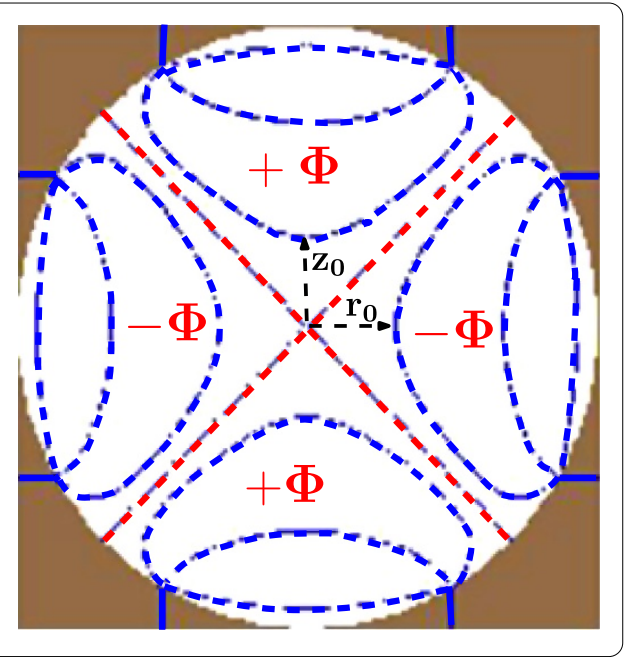

Here $r_{0}$ is the distance from the rod surface to the $z$ axis. These equations are forms of Mathieu's differential equation [6-9]. The solutions of this equation are complex and are discussed in the literature [10]. The solutions are classified as stable and unstable. If the amplitude of oscillatory motion is less or equal to one, the charged particle does not strike the rods and then its trajectory is stable. When the amplitude of a particle trajectory is larger than one, then the particle motion is mathematically unstable.

\section{Preliminaries of the fuzzy sets}

In this section we prepare some definitions and properties of fuzzy sets [11-17].

Fuzzy sets: According to Zadeh (1965), a fuzzy set is a generalization of a classical set that allows a membership function to take any value in the unit interval $[0,1]$. The formal definition of a fuzzy set [16] is as follows.

Definition 1 Let $\Omega$ be a universal set. A fuzzy set $A$ in $\Omega$ is defined by a membership function $A(x)$ that maps every element in $\Omega$ to the unit interval $[0,1]$. A fuzzy set $A$ in $\Omega$ may also be presented as a set of ordered pairs of a generic element $x$ and its membership value, as shown in the following equation:

$$
A=\{(x, A(x)) \mid x \in \Omega\} .
$$

Definition 2 Let $A$ be a fuzzy interval defined in $R$. The $\alpha$-cut of $A$ is the crisp set $[A]^{0}$ that contains all elements in $R$ such that the membership values of $A$ is greater than or equal to $a$, that is,

$$
[A]^{\alpha}=\{x \in R \mid A(x) \geq \alpha\}, \quad \alpha \in(0,1]
$$

For a fuzzy interval $A$, its $\alpha$-cuts are closed intervals in $R$, and we denote them by

$$
[A]^{\alpha}=\left[a_{1}^{\alpha}, a_{2}^{\alpha}\right], \quad \alpha \in(0,1] .
$$


Definition 3 A fuzzy interval $A$ is called equipossible fuzzy if it is fully determined by the pair $(a, b)$ of crisp numbers with $a<b$ and its membership function is given by

$$
A(x)= \begin{cases}1 ; & \text { if } a \leq x \leq b \\ 0 ; & \text { otherwise }\end{cases}
$$

Definition 4 A fuzzy interval $A$ is called triangular fuzzy if it is fully determined by the triplet $(a, b, c)$ of crisp numbers with $a<b<c$ and its membership function is given by

$$
A(x)= \begin{cases}\frac{x-a}{b-a} ; & \text { if } a \leq x \leq b \\ \frac{x-c}{b-c} ; & \text { if } b \leq x \leq c \\ 0 ; & \text { otherwise. }\end{cases}
$$

Definition 5 Let $f:(a, b) \rightarrow E$ and $x_{0} \in(a, b)$. We say that $f$ is strongly generalized $H$-differentiable at $x_{0}$, if there exists an element $f^{\prime}\left(x_{0}\right) \in E$ such that

(1) for all $h>0$ sufficiently near to $0, \exists f\left(x_{0}+h\right) \ominus f\left(x_{0}\right), \exists f\left(x_{0}\right) \ominus f\left(x_{0}-h\right)$ and the limits

$$
\lim _{h \rightarrow 0^{+}} \frac{f\left(x_{0}+h\right) \ominus f\left(x_{0}\right)}{h}=\lim _{h \rightarrow 0^{+}} \frac{f\left(x_{0}\right) \ominus f\left(x_{0}-h\right)}{h}=f^{\prime}\left(x_{0}\right)
$$

or

(2) for all $h<0$ sufficiently near to $0, \exists f\left(x_{0}+h\right) \ominus f\left(x_{0}\right), \exists f\left(x_{0}\right) \ominus f\left(x_{0}-h\right)$ and the limits

$$
\lim _{h \rightarrow 0^{-}} \frac{f\left(x_{0}\right) \ominus f\left(x_{0}+h\right)}{h}=\lim _{h \rightarrow 0^{-}} \frac{f\left(x_{0}-h\right) \ominus f\left(x_{0}\right)}{h}=f^{\prime}\left(x_{0}\right) .
$$

In the special case when $f$ is a fuzzy-valued function, we have the following result.

Theorem 1 [17] Let $f: R \rightarrow E$ be a function and denote $f(t)=(\bar{f}(t, r), f(t, r))$ for each $r \in$ $[0,1]$. Then

- iff is differentiable in the first form (1) in Definition 5 , then $\bar{f}(t, r)$ and $f(t, r)$ are differentiable functions and $f^{\prime}(t)=\left(\bar{f}^{\prime}(t, r), f^{\prime}(t, r)\right)$,

- iff is differentiable in the second form (2) in Definition 5, then $\bar{f}(t, r)$ and $f(t, r)$ are differentiable functions and $f^{\prime}(t)=\left(\bar{f}^{\prime}(t, r), f_{-}^{\prime}(t, r)\right)$.

\section{The quadrupole mass filter motion equations with fuzzy initial values}

In this section we use the presented new algorithm for solving the equations of motion for a singly charged positive ion in the QMF by fuzzy initial values.

We consider the equations of motion in the QMF with fuzzy initial values as follows:

$$
\begin{array}{lcr}
z^{\prime \prime}(\xi)=-\left(a_{z}-2 q_{z} \cos 2 \xi\right) z ; & \tilde{z}\left(\xi_{0}\right)=\tilde{z}_{0}, & \tilde{z}^{\prime}\left(\xi_{0}\right)=\tilde{z}_{0}^{\prime}, \\
r^{\prime \prime}(\xi)=\left(a_{r}-2 q_{r} \cos 2 \xi\right) r ; & \tilde{r}\left(\xi_{0}\right)=\tilde{r}_{0}, & \tilde{r}^{\prime}\left(\xi_{0}\right)=\tilde{r}_{0}^{\prime},
\end{array}
$$

where $z, r$ are fuzzy functions of $\xi$ and $\tilde{z}\left(\xi_{0}\right)=\tilde{z}_{0}, \tilde{z}^{\prime}\left(\xi_{0}\right)=\tilde{z}_{0}^{\prime}$ and $\tilde{r}\left(\xi_{0}\right)=\tilde{r}_{0}, \tilde{r}^{\prime}\left(\xi_{0}\right)=\tilde{r}_{0}^{\prime}$ are trapezoidal or trapezoidal shaped fuzzy numbers. 
We denote the fuzzy functions $z$ and $r$ by $z=[\bar{z}, \underline{z}]$ and $r=[\bar{r}, \underline{r}]$, respectively. It means that the $\alpha$-level sets of $z(\xi)$ and $r(\xi)$ for $\xi \in\left[\xi_{0}, T\right]$ are

$$
\begin{array}{ll}
{[z(\xi)]_{\alpha}=[\bar{z}(\xi ; \alpha), \underline{z}(\xi ; \alpha)],} & {\left[z\left(\xi_{0}\right)\right]_{\alpha}=\left[\bar{z}\left(\xi_{0} ; \alpha\right), \underline{z}\left(\xi_{0} ; \alpha\right)\right],} \\
{\left[r\left(\xi_{0}\right)\right]_{\alpha}=\left[\bar{r}\left(\xi_{0} ; \alpha\right), \underline{r}\left(\xi_{0} ; \alpha\right)\right],} & {[r(\xi)]_{\alpha}=[\bar{r}(\xi ; \alpha), \underline{r}(\xi ; \alpha)],} \\
{\left[z^{\prime}\left(\xi_{0}\right)\right]_{\alpha}=\left[\bar{z}^{\prime}\left(\xi_{0} ; \alpha\right), \underline{z}^{\prime}\left(\xi_{0} ; \alpha\right)\right],} & {\left[r^{\prime}\left(\xi_{0}\right)\right]_{\alpha}=\left[\bar{r}^{\prime}\left(\xi_{0} ; \alpha\right), \underline{r}^{\prime}\left(\xi_{0} ; \alpha\right)\right]}
\end{array}
$$

with $\alpha \in(0,1]$.

\section{Fuzzy initial value problems}

In this section, we first consider the following second-order ordinary differential equation with fuzzy initial values [11-20]:

$$
\begin{aligned}
& y^{\prime \prime}(t)=f\left(t, y(t), y^{\prime}(t)\right), \quad t \in[0, T], \\
& \tilde{y}\left(t_{0}\right)=\tilde{y}_{0}, \quad \tilde{y}^{\prime}\left(t_{0}\right)=\tilde{y}_{0}^{\prime},
\end{aligned}
$$

where $y$ is a fuzzy function of $t, f\left(t, y, y^{\prime}\right)$ is a fuzzy function of the crisp variable $t$ and the fuzzy variable $y, y^{\prime} ; y^{\prime \prime}$ is the second fuzzy derivative of $y$ and $\tilde{y}\left(t_{0}\right)=\tilde{y}_{0}, \tilde{y}^{\prime}\left(t_{0}\right)=\tilde{y}_{0}^{\prime}$ are trapezoidal or trapezoidal shaped fuzzy numbers.

We denote the fuzzy function $y$ by $y=[\bar{y}, y]$. It means that the $\alpha$-level sets of $y(t)$ for $t \in\left[t_{0}, T\right]$ are

$$
\begin{aligned}
& {[y(t)]_{\alpha}=[\bar{y}(t ; \alpha), \underline{y}(t ; \alpha)],} \\
& {\left[y\left(t_{0}\right)\right]_{\alpha}=\left[\bar{y}\left(t_{0} ; \alpha\right), \underline{y}\left(t_{0} ; \alpha\right)\right],} \\
& {\left[y^{\prime}\left(t_{0}\right)\right]_{\alpha}=\left[\bar{y}^{\prime}\left(t_{0} ; \alpha\right), \underline{y^{\prime}}\left(t_{0} ; \alpha\right)\right]}
\end{aligned}
$$

with $\alpha \in(0,1]$.

\section{Formulation of a new algorithm for solving second-order ordinary differential equations}

Consider a second-order initial value problem of the form

$$
\begin{aligned}
& y^{\prime \prime}(t)=f\left(t, y, y^{\prime}(t)\right)+g(t)+K ; \quad n=1,2, \ldots, \\
& y(0)=c_{0}, \quad y^{\prime}(0)=c_{1} .
\end{aligned}
$$

Here, $y(0)=c_{0}, y^{\prime}(0)=c_{1}$ are called the initial conditions, while $K$ is constant.

This was turned into an iterative scheme by substituting a guess $\Psi_{m}(t)(m=0,1,2,3, \ldots)$ for the value of $y(t)$ on the right, and the result as the definition of $\Psi_{m}(t)$, which substitutes for the value of $y(t)$ on the left. The new algorithm was defined to compute the sequence $\left\{\Psi_{m}\right\}_{m=0}^{+\infty}$ as follows:

$$
\begin{aligned}
& \Psi_{0}=y\left(t_{0}\right) ; \\
& \Psi_{1}=\int_{t_{0}}^{t} y^{\prime}\left(t_{0}\right) d t+\int_{t_{0}}^{t} \int_{t_{0}}^{t}\left[f\left(t, y, y^{\prime}(t)\right)+g(t)+K\right] d t d t ;
\end{aligned}
$$




$$
\Psi_{m}=\int_{t_{0}}^{t} \int_{t_{0}}^{t} f\left(t, \Psi_{m-1}, \Psi_{m-1}^{\prime}\right) d t d t \quad(m>1) .
$$

The answer of Eq. (21), $y(t)$, is defined as approximated by the following series:

$$
y(t) \simeq \tilde{y}(t)=\sum_{i=0}^{M} \Psi_{i}
$$

Here, $M$ is the order of approximation and $\tilde{y}$ is the approximate answer for Eq. (21).

\section{To solve quadrupole mass filter systems}

In this section we solve Eqs. (11) and (12) with the fuzzy initial values by a proposed algorithm as follows:

$$
\begin{aligned}
& {\left[z\left(\xi_{0}\right)\right]_{\alpha}=\left[\bar{z}\left(\xi_{0} ; \alpha\right), \underline{z}\left(\xi_{0} ; \alpha\right)\right]=\left[c_{1}-a_{1}+a_{1} \alpha, c_{1}+a_{1}-a_{1} \alpha\right],} \\
& {\left[z^{\prime}\left(\xi_{0}\right)\right]_{\alpha}=\left[\bar{z}^{\prime}\left(\xi_{0} ; \alpha\right), \underline{z}^{\prime}\left(\xi_{0} ; \alpha\right)\right]=\left[-a_{1}+a_{1} \alpha, a_{1}-a_{1} \alpha\right],} \\
& {\left[r\left(\xi_{0}\right)\right]_{\alpha}=\left[\bar{r}\left(\xi_{0} ; \alpha\right), \underline{r}\left(\xi_{0} ; \alpha\right)\right]=\left[c_{2}-a_{2}+a_{2} \alpha, c_{2}+a_{2}-a_{2} \alpha\right],} \\
& {\left[r^{\prime}\left(\xi_{0}\right)\right]_{\alpha}=\left[\bar{r}^{\prime}\left(\xi_{0} ; \alpha\right), \underline{r}^{\prime}\left(\xi_{0} ; \alpha\right)\right]=\left[-a_{2}+a_{2} \alpha, a_{2}-a_{2} \alpha\right] .}
\end{aligned}
$$

With this fuzzy initial values the exact solutions (see Appendix 1) are as follows:

$$
\begin{aligned}
& \bar{z}(\xi ; \alpha)=\left(c_{1}-a_{1}+a_{1} \alpha\right) \text { MathieuC }\left(a_{z}, q_{z}, \xi\right)+\left(-a_{1}+a_{1} \alpha\right) \text { MathieuS }\left(a_{z}, q_{z}, \xi\right), \\
& \underline{z}(\xi ; \alpha)=\left(c_{1}+a_{1}-a_{1} \alpha\right) \text { MathieuC }\left(a_{z}, q_{z}, \xi\right)+\left(a_{1}-a_{1} \alpha\right) \text { MathieuS }\left(a_{z}, q_{z}, \xi\right), \\
& \bar{r}(\xi ; \alpha)=\left(c_{2}-a_{2}+a_{2} \alpha\right) \text { MathieuC }\left(a_{r}, q_{r}, \xi\right)+\left(-a_{2}+a_{2} \alpha\right) \text { MathieuS }\left(a_{r}, q_{r}, \xi\right), \\
& \underline{r}(\xi ; \alpha)=\left(c_{2}+a_{2}-a_{2} \alpha\right) \text { MathieuC }\left(a_{r}, q_{r}, \xi\right)+\left(a_{2}-a_{2} \alpha\right) \text { MathieuS }\left(a_{r}, q_{r}, \xi\right) .
\end{aligned}
$$

Here MathieuC and MathieuS are even and odd functions of $\xi$, respectively; and $c_{1}=z(0)$, $c_{2}=r(0)$ are initial conditions and $a_{1}, a_{2}$ are constant. Now, by using Eqs. (22) up to (24), the $m$ th-order solution of Eqs. (11) and (12) with initials conditions (26) up to (29) is as follows:

$$
\begin{aligned}
& {\left[\Psi_{\bar{z}, 0}, \Psi_{\underline{z}, 0}\right]=c_{1}-a_{1}+a_{1} \alpha,} \\
& {\left[\Psi_{\bar{z}, 1}, \Psi_{\underline{z}, 1}\right]=\int_{\xi_{0}}^{\xi}\left[\bar{z}\left(\xi_{0} ; \alpha\right), \underline{z}\left(\xi_{0} ; \alpha\right)\right] d \xi-\int_{\xi_{0}}^{\xi} \int_{\xi_{0}}^{\xi}\left(a_{z}-q_{z} \cos (2 \xi)\right)[\bar{z}(\xi ; \alpha), \underline{z}(\xi ; \alpha)] d \xi d \xi,} \\
& {\left[\Psi_{\bar{z}, m}, \Psi_{\underline{z}, m}\right]=\int_{\xi_{0}}^{\xi} \int_{\xi_{0}}^{\xi}\left(a_{z}-q_{z} \cos (2 \xi)\right)\left[\Psi_{\bar{z}, m-1}, \Psi_{\underline{z}, m-1}\right] d \xi d \xi \quad(m>1),} \\
& {\left[\Psi_{\bar{r}, 0}, \Psi_{\underline{r}, 0}\right]=c_{2}-a_{2}+a_{2} \alpha,} \\
& {\left[\Psi_{\bar{r}, 1}, \Psi_{\underline{r}, 1}\right]=\int_{\xi_{0}}^{\xi}\left[\bar{r}\left(\xi_{0} ; \alpha\right), \underline{r}\left(\xi_{0} ; \alpha\right)\right] d \xi+\int_{\xi_{0}}^{\xi} \int_{\xi_{0}}^{\xi}\left(a_{r}-q_{r} \cos (2 \xi)\right)[\bar{r}(\xi ; \alpha), \underline{r}(\xi ; \alpha)] d \xi d \xi} \\
& {\left[\Psi_{\bar{r}, m}, \Psi_{\underline{r}, m}\right]=\int_{\xi_{0}}^{\xi} \int_{\xi_{0}}^{\xi}\left(a_{r}-q_{r} \cos (2 \xi)\right)\left[\Psi_{\bar{r}, m-1}, \Psi_{\underline{r}, m-1}\right] d \xi d \xi \quad(m>1),}
\end{aligned}
$$


which can be easily solved by symbolic computation software such as Matlab, Maple and Mathematica. After finding $\Psi_{\bar{z}, m}, \Psi_{\underline{z}, m}$ and $\Psi_{\bar{r}, m}, \Psi_{\underline{r}, m}$, we have

$$
\begin{aligned}
& {[\bar{z}(\xi ; \alpha), \underline{z}(\xi ; \alpha)] \simeq \sum_{m=0}^{M}\left[\Psi_{\bar{z}, m}, \Psi_{\underline{z}, m}\right],} \\
& {[\bar{r}(\xi ; \alpha), \underline{r}(\xi ; \alpha)] \simeq \sum_{m=0}^{M}\left[\Psi_{\bar{r}, m}, \Psi_{\underline{r}, m}\right] .}
\end{aligned}
$$

Here, $M$ is the order of approximation. By Maple software (see Appendix 2), we now successively obtain

$$
\begin{aligned}
& \bar{z}(\xi ; \alpha)=c_{1}-a_{1}+a_{1} \alpha+\left(-a_{1}+a_{1} \alpha\right) \xi+\left(-\frac{1}{2} a_{z} a_{1}-q_{z} a_{1} \alpha-q_{z} c_{1}+q_{z} a_{1}\right. \\
& \left.+\frac{1}{2} a_{z} a_{1} \alpha+\frac{1}{2} a_{z} c_{1}\right) \xi^{2}+\left(-\frac{1}{6} a_{z} a_{1}-\frac{1}{3} q_{z} a_{1} \alpha+\frac{1}{3} q_{z} a_{1}+\frac{1}{6} a_{z} a_{1} \alpha\right) \xi^{3} \\
& +\left(-\frac{1}{6} q_{z}^{2} a_{1}+\frac{1}{6} q_{z}^{2} c_{1}+\frac{1}{3} q_{z} a_{1} \alpha-\frac{1}{24} a_{z}^{2} a_{1}+\frac{1}{6} a_{z} a_{1} q_{z}-\frac{1}{6} a_{z} c_{1} q_{z}\right. \\
& \left.+\frac{1}{6} q_{z}^{2} a_{1} \alpha+\frac{1}{3} q_{z} c_{1}-\frac{1}{3} q_{z} a_{1}+\frac{1}{24} a_{z}^{2} a_{1} \alpha-\frac{1}{6} a_{z} a_{1} \alpha q_{z}+\frac{1}{24} a_{z}^{2} c_{1}\right) \xi^{4} \\
& +\left(-\frac{1}{30} q_{z}^{2} a_{1}+\frac{1}{5} q_{z} a_{1} \alpha-\frac{1}{120} a_{z}^{2} a_{1}+\frac{1}{30} a_{z} a_{1} q_{z}+\frac{1}{30} q_{z}^{2} a_{1} \alpha-\frac{1}{5} q_{z} a_{1}\right. \\
& \left.+\frac{1}{120} a_{z}^{2} a_{1} \alpha-\frac{1}{30} a_{z} a_{1} \alpha q_{z}\right) \xi^{5}+\cdots \\
& =\left(c_{1}-a_{1}+a_{1} \alpha\right) \text { MathieuC }\left(a_{z}, q_{z}, \xi\right)+\left(-a_{1}+a_{1} \alpha\right) \text { MathieuS }\left(a_{z}, q_{z}, \xi\right) \text {, } \\
& \underline{z}(\xi ; \alpha)=c_{1}+a_{1}-a_{1} \alpha+\left(a_{1}-a_{1} \alpha\right) \xi+\left(\frac{1}{2} a_{z} a_{1}+q_{z} a_{1} \alpha-q_{z} c_{1}-q_{z} a_{1}\right. \\
& \left.-\frac{1}{2} a_{z} a_{1} \alpha+\frac{1}{2} a_{z} c_{1}\right) \xi^{2}+\left(\frac{1}{6} a_{z} a_{1}+\frac{1}{3} q_{z} a_{1} \alpha-\frac{1}{3} q_{z} a_{1}-\frac{1}{6} a_{z} a_{1} \alpha\right) \xi^{3} \\
& +\left(\frac{1}{6} q_{z}^{2} a_{1}+\frac{1}{6} q_{z}^{2} c_{1}-\frac{1}{3} q_{z} a_{1} \alpha+\frac{1}{24} a_{z}^{2} a_{1}-\frac{1}{6} a_{z} a_{1} q_{z}-\frac{1}{6} a_{z} c_{1} q_{z}\right. \\
& \left.-\frac{1}{6} q_{z}^{2} a_{1} \alpha+\frac{1}{3} q_{z} c_{1}+\frac{1}{3} q_{z} a_{1}-\frac{1}{24} a_{z}^{2} a_{1} \alpha+\frac{1}{6} a_{z} a_{1} \alpha q_{z}+\frac{1}{24} a_{z}^{2} c_{1}\right) \xi^{4} \\
& +\left(\frac{1}{30} q_{z}^{2} a_{1}-\frac{1}{5} q_{z} a_{1} \alpha+\frac{1}{120} a_{z}^{2} a_{1}-\frac{1}{30} a_{z} a_{1} q_{z}-\frac{1}{30} q_{z}^{2} a_{1} \alpha+\frac{1}{5} q_{z} a_{1}\right. \\
& \left.-\frac{1}{120} a_{z}^{2} a_{1} \alpha+\frac{1}{30} a_{z} a_{1} \alpha q_{z}\right) \xi^{5}+\cdots \\
& =\left(c_{1}+a_{1}-a_{1} \alpha\right) \text { MathieuC }\left(a_{z}, q_{z}, \xi\right)+\left(a_{1}-a_{1} \alpha\right) \text { MathieuS }\left(a_{z}, q_{z}, \xi\right), \\
& \bar{r}(t ; \alpha)=c_{2}-a_{2}+a_{2} \alpha+\left(-a_{2}+a_{2} \alpha\right) \xi+\left(\frac{1}{2} a_{r} a_{2}+q_{r} a_{2} \alpha+q_{r} c_{2}-q_{r} a_{2}\right. \\
& \left.-\frac{1}{2} a_{r} a_{2} \alpha-\frac{1}{2} a_{r} c_{2}\right) \xi^{2}+\left(\frac{1}{6} a_{r} a_{2}+\frac{1}{3} q_{r} a_{2} \alpha-\frac{1}{3} q_{r} a_{2}-\frac{1}{6} a_{r} a_{2} \alpha\right) \xi^{3} \\
& +\left(-\frac{1}{6} q_{r}^{2} a_{2}+\frac{1}{6} q_{r}^{2} c_{2}-\frac{1}{3} q_{r} a_{2} \alpha-\frac{1}{24} a_{r}^{2} a_{2}+\frac{1}{6} a_{r} a_{2} q_{r}-\frac{1}{6} a_{r} c_{2} q_{r}\right.
\end{aligned}
$$




$$
\begin{aligned}
& \left.+\frac{1}{6} q_{r}^{2} a_{2} \alpha-\frac{1}{3} q_{r} c_{2}+\frac{1}{3} q_{r} a_{2}+\frac{1}{24} a_{r}^{2} a_{2} \alpha-\frac{1}{6} a_{r} a_{2} \alpha q_{r}+\frac{1}{24} a_{r}^{2} c_{2}\right) \xi^{4} \\
& +\left(-\frac{1}{30} q_{r}^{2} a_{2}-\frac{1}{5} q_{r} a_{2} \alpha-\frac{1}{120} a_{r}^{2} a_{2}+\frac{1}{30} a_{r} a_{2} q_{r}-\frac{1}{30} q_{r}^{2} a_{1} \alpha+\frac{1}{5} q_{r} a_{2}\right. \\
& \left.+\frac{1}{120} a_{r}^{2} a_{2} \alpha-\frac{1}{30} a_{r} a_{2} \alpha q_{r}\right) \xi^{5}+\cdots \\
& =\left(c_{2}-a_{2}+a_{2} \alpha\right) \text { MathieuC }\left(a_{r}, q_{r}, \xi\right)+\left(-a_{2}+a_{2} \alpha\right) \text { MathieuS }\left(a_{r}, q_{r}, \xi\right) \text {, } \\
& \underline{r}(\xi ; \alpha)=c_{2}+a_{2}-a_{2} \alpha+\left(a_{2}-a_{2} \alpha\right) \xi+\left(\frac{1}{2} a_{r} a_{1}-q_{r} a_{2} \alpha+q_{r} c_{2}+q_{r} a_{2}\right. \\
& \left.+\frac{1}{2} a_{r} a_{2} \alpha-\frac{1}{2} a_{r} c_{2}\right) \xi^{2}+\left(-\frac{1}{6} a_{r} a_{2}-\frac{1}{3} q_{r} a_{2} \alpha+\frac{1}{3} q_{r} a_{2}+\frac{1}{6} a_{r} a_{2} \alpha\right) \xi^{3} \\
& +\left(\frac{1}{6} q_{r}^{2} a_{2}+\frac{1}{6} q_{r}^{2} c_{2}+\frac{1}{3} q_{r} a_{2} \alpha+\frac{1}{24} a_{r}^{2} a_{2}-\frac{1}{6} a_{r} a_{2} q_{r}-\frac{1}{6} a_{r} c_{2} q_{r}\right. \\
& \left.-\frac{1}{6} q_{r}^{2} a_{2} \alpha-\frac{1}{3} q_{r} c_{2}-\frac{1}{3} q_{r} a_{2}-\frac{1}{24} a_{r}^{2} a_{2} \alpha+\frac{1}{6} a_{r} a_{2} \alpha q_{r}+\frac{1}{24} a_{r}^{2} c_{1}\right) \xi^{4} \\
& +\left(\frac{1}{30} q_{r}^{2} a_{2}+\frac{1}{5} q_{r} a_{2} \alpha+\frac{1}{120} a_{r}^{2} a_{2}-\frac{1}{30} a_{r} a_{2} q_{r}-\frac{1}{30} q_{r}^{2} a_{2} \alpha-\frac{1}{5} q_{r} a_{2}\right. \\
& \left.-\frac{1}{120} a_{r}^{2} a_{2} \alpha+\frac{1}{30} a_{r} a_{2} \alpha q_{r}\right) \xi^{5}+\cdots \\
& =\left(c_{2}+a_{2}-a_{2} \alpha\right) \text { MathieuC }\left(a_{r}, q_{r}, \xi\right)+\left(a_{2}-a_{2} \alpha\right) \text { MathieuS }\left(a_{r}, q_{r}, \xi\right) \text {. }
\end{aligned}
$$

\section{Numerical results}

In this section we bring some numerical results for illustration (see Appendix 3). Initial parameters for these numerical results have been used as follows:

$$
\begin{aligned}
& c_{1}=z(0)=0.5 ; \quad c_{2}=r(0)=0.5 ; \\
& z^{\prime}(0)=0 ; \quad r^{\prime}(0)=0 ; \quad a_{1}=a_{2}=0.1 ; \\
& a_{z}=-a_{r}=0 ; \quad q_{z}=-q_{r}=0.4 .
\end{aligned}
$$

Figure 2(a) and (b) shows the approximate solutions with $M=20,(\mathrm{a}): \bar{z}(\xi, \alpha), \underline{z}(\xi, \alpha)$, (b): $\bar{r}(\xi, \alpha), \underline{r}(\xi, \alpha)$, gold color: $\bar{z}(\xi, \alpha), \bar{r}(\xi, \alpha)$ and green color: $\underline{z}(\xi, \alpha), \underline{r}(\xi, \alpha)$. Figure 2(a) and (b) shows that for $\alpha=1$ we have $z(\xi)=\bar{z}(\xi, \alpha)=\underline{z}(\xi, \alpha)$ and $r(\xi)=\bar{r}(\xi, \alpha)=\underline{r}(\xi, \alpha)$. Figure 3 shows the $\alpha$-discretization of a fuzzy interval, blue color: $\bar{z}(2, \alpha), \underline{z}(2, \alpha)$ and red color: $\bar{r}(2, \alpha), \underline{r}(2, \alpha)$. Figure $4(\mathrm{a})$ and (b) shows the ion trajectories in real time for $a_{z}=a_{r}=0$, $q_{z}=-q_{r}=0.4$ and $M=20,(\mathrm{a}): \bar{z}(\xi, 0.8), \underline{z}(\xi, 0.8),(\mathrm{b}): \bar{r}(\xi, 0.8), \underline{r}(\xi, 0.8)$, blue line: $\bar{z}, \bar{r}$ and red line: $\underline{z}, \underline{r}$. Figure 5 shows the ion trajectory in $\bar{z}-\bar{r}$ and $\underline{z}-\underline{r}$ plans when $a_{z}=-a_{r}=0$, $q_{z}=-q_{r}=0.4$ and $M=20$, blue color (solid line): $\bar{z}(\xi, 0.8)-\bar{z}(\xi, 0.8)$ and red color (dash line): $\underline{z}(\xi, 0.8)-\underline{z}(\xi, 0.8)$.

\section{Discussion and conclusion}

We have solved fuzzy differential equations of a quadrupole mass filter with fuzzy initial conditions by a proposed new algorithm. The results obtained show that fuzzy solution is compatible to an exact solution. For the illustration, the numerical results with order 20 $(M=20)$ have been presented. Figure $2(\mathrm{a})$ and (b) is the approximate solution with $M=20$ 

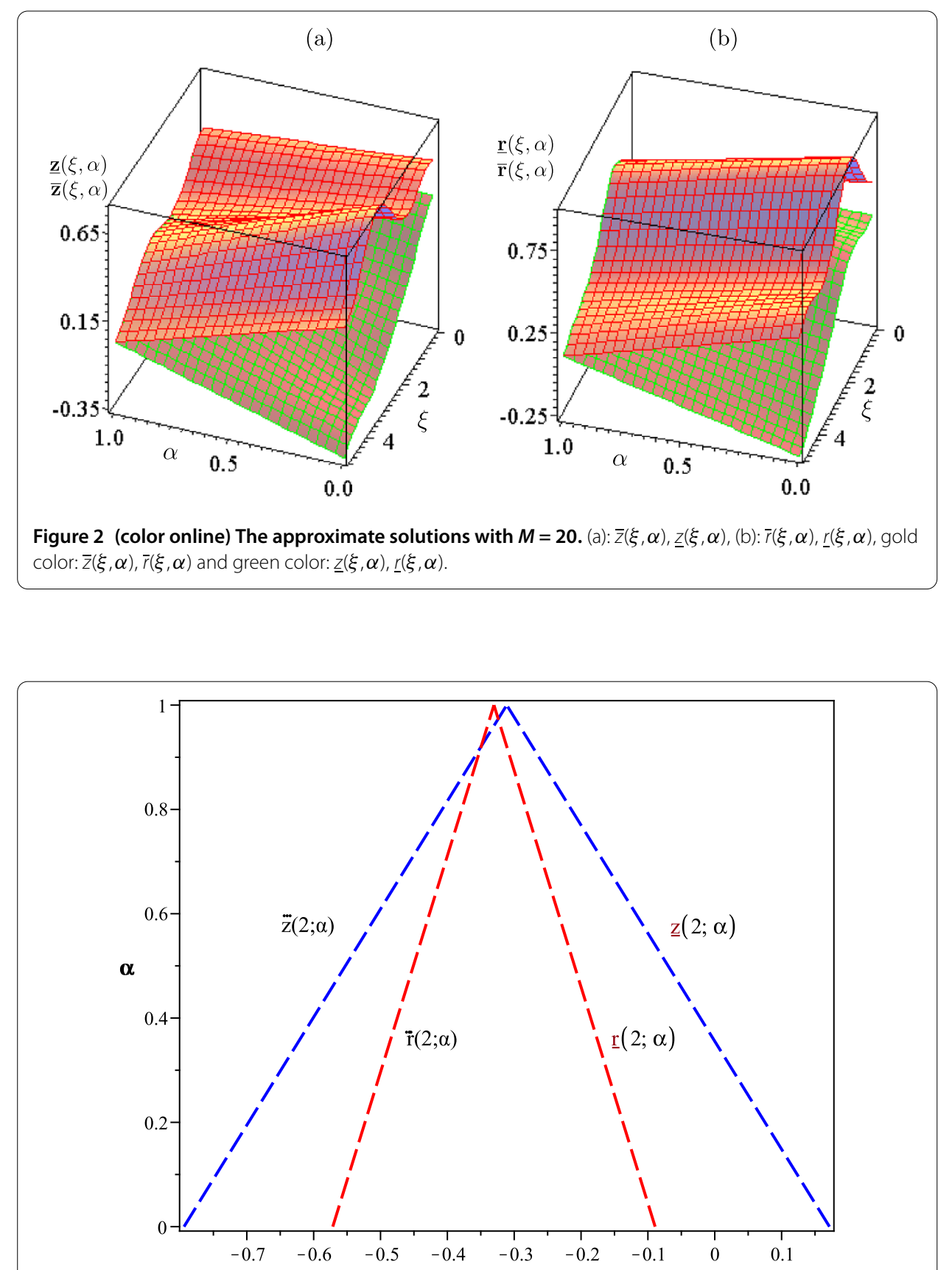

Figure 3 (color online) $\alpha$-discretization of a fuzzy interval, blue color: $\bar{z}(2, \alpha), \underline{z}(2, \alpha)$ and red color: $\bar{r}(2, \alpha), \underline{r}(2, \alpha)$.

for $z$ and $r$, respectively. In this figure, for $\alpha=1$ we have found that $z(\xi)=\bar{z}(\xi, \alpha)=\underline{z}(\xi, \alpha)$ and $r(\xi)=\bar{r}(\xi, \alpha)=\underline{r}(\xi, \alpha)$, respectively. Therefore, probability $\alpha=1$ gives the normal answer for a quadrupole mass filter without fuzzy initial conditions. The method showed a potential application to solve complicated linear and nonlinear differential equations in a quadrupole field, especially in fine tuning accelerators and, generally speaking, in physics of high energy. 

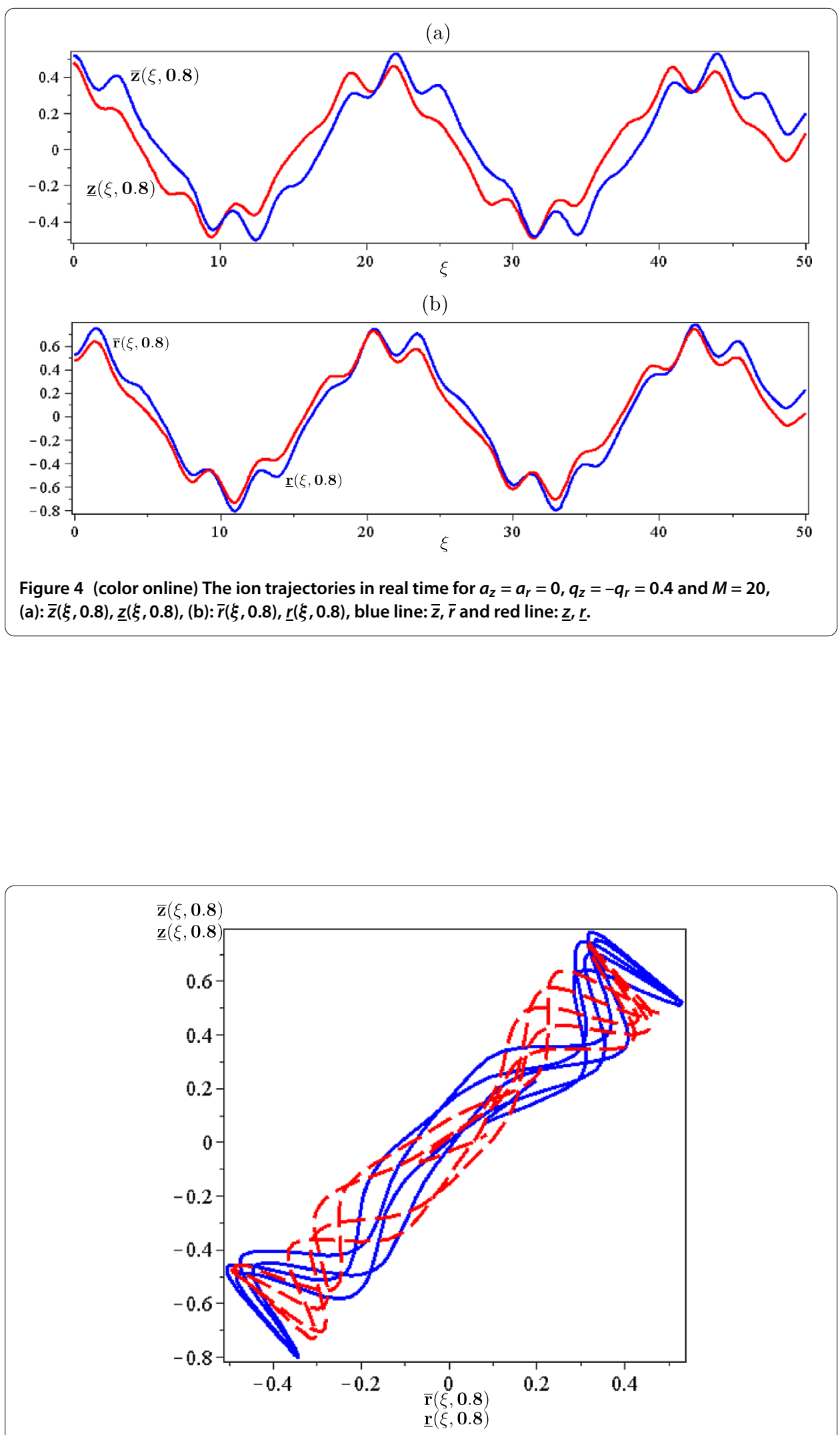

Figure 5 (color online) The ion trajectory in $\bar{z}-\bar{r}$ and $\underline{z}-\underline{r}$ plans when $a_{z}=a_{r}=0, q_{z}=-q_{r}=0.4$ and $M=20$, blue color (solid line): $\bar{z}(\xi, 0.8)-\bar{z}(\xi, 0.8)$ and red color (dash line): $\underline{z}(\xi, 0.8)-\underline{z}(\xi, 0.8)$. 


\section{Appendix 1}

\section{Maple code for deriving the analytical solutions:}

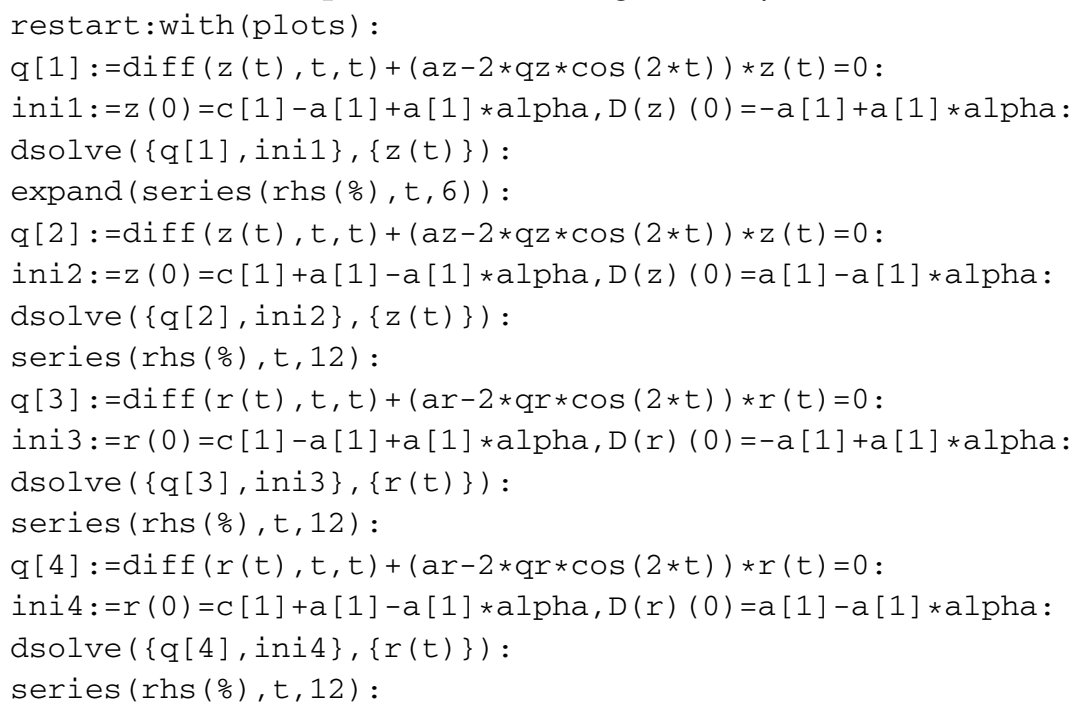

\section{Appendix 2}

Maple code for deriving the analytical solutions by the new proposed algorithm: restart: with (plots) $: \mathrm{N}:=30:$ alpha: $=0.8$ : $\mathrm{x} 1[0]:=0.4+0.1 * \mathrm{alpha}: \mathrm{dx} 1[0]:=-0.1+0.1 * \mathrm{alpha}:$ $\mathrm{x} 2[0]:=0.6-0.1 * a 1 \mathrm{pha}: \mathrm{dx} 2[0]:=0.1-0.1 * a 1 \mathrm{pha}:$ $\mathrm{y} 1[0]:=0.4+0.1 * \mathrm{alpha}: \operatorname{dy} 1[0]:=-0.1+0.1 * \mathrm{alpha}:$ y2 $[0]:=0.6-0.1 * a l p h a:$ dy2 $[0]:=0.1-0.1 * a l p h a: h:=1.0: k:=0.0: F[1]:=1:$ $\mathrm{a}[\mathrm{x}]:=0: \mathrm{a}[\mathrm{y}]:=0: \mathrm{q}[\mathrm{x}]:=0.4: \mathrm{q}[\mathrm{y}]:=0.4: \mathrm{x} 1[1]:=x 1[0]:$ $x 1[2]:=\operatorname{int}(d x 1[0], t=0 \ldots t)+h * \operatorname{int}(\operatorname{int}((a[x]-2 * q[x]$ $* \cos (2 * t) /(1-k * \cos (4 * t))) *(x 1[1]), t=0 \ldots t), t=0 \ldots t): x 2[1]:=x 2[0]:$ $\mathrm{x} 2[2]:=\operatorname{int}(\mathrm{dx} 2[0], \mathrm{t}=0 \ldots \mathrm{t})+\mathrm{h} * \operatorname{int}(\operatorname{int}((\mathrm{a}[\mathrm{x}]-2 * \mathrm{q}[\mathrm{x}]$ $* \cos (2 * t) /(1-k * \cos (4 * t))) *(x 2[1]), t=0 \ldots t), t=0 \ldots t): y 1[1]:=y 1[0]:$ $\mathrm{y} 1[2]:=\operatorname{int}(\mathrm{dy} 1[0], \mathrm{t}=0 . \mathrm{t})-\operatorname{int}(\operatorname{int}((\mathrm{a}[\mathrm{y}]-2 * \mathrm{q}[\mathrm{y}]$ $* \cos (2 * t) /(1-k * \cos (4 * t))) *(y 1[1]), t=0 \ldots t), t=0 \ldots t): y 2[1]:=y 2[0]:$ $\mathrm{y} 2[2]:=\operatorname{int}(\mathrm{dy} 2[0], \mathrm{t}=0 \ldots \mathrm{t})-\operatorname{int}(\operatorname{int}((\mathrm{a}[\mathrm{y}]-2 * \mathrm{q}[\mathrm{y}]$ $* \cos (2 * t) /(1-k * \cos (4 * t))) *(y 2[1]), t=0 \ldots t), t=0 \ldots t)$ : for $m$ from 3 by 1 to $\mathrm{N}$ do $\mathrm{x} 1[\mathrm{~m}]:=\mathrm{h}^{\wedge}(\mathrm{m}-1) * \operatorname{int}(\operatorname{int}((\mathrm{a}[\mathrm{x}]-2 * \mathrm{q}[\mathrm{x}] * \cos (2 * \mathrm{t}) /$ $(1-\mathrm{k} * \cos (4 * t))) *(\mathrm{x} 1[\mathrm{~m}-1]), \mathrm{t}=0 \ldots \mathrm{t}), \mathrm{t}=0 \ldots \mathrm{t}):$ $\mathrm{x} 2[\mathrm{~m}]:=\mathrm{h} \wedge(\mathrm{m}-1) * \operatorname{int}(\operatorname{int}((\mathrm{a}[\mathrm{x}]-2 * \mathrm{q}[\mathrm{x}] * \cos (2 * \mathrm{t}) /$ $(1-\mathrm{k} * \cos (4 * t))) *(x 2[m-1]), t=0 \ldots t), t=0 \ldots t):$ $\mathrm{y} 1[\mathrm{~m}]:=-\operatorname{int}(\operatorname{int}((\mathrm{a}[\mathrm{y}]-2 * \mathrm{q}[\mathrm{y}] * \cos (2 * \mathrm{t}) /$ $(1-k * \cos (4 * t))) *(y 1[m-1]), t=0 \ldots t), t=0 \ldots t):$ $\mathrm{y} 2[\mathrm{~m}]:=-\operatorname{int}(\operatorname{int}((\mathrm{a}[\mathrm{y}]-2 * \mathrm{q}[\mathrm{y}] * \cos (2 * \mathrm{t}) /$ $(1-k * \cos (4 * t))) *(y 2[m-1]), t=0 \ldots t)$ $, t=0 \ldots t)$ : end do: $x:=\operatorname{sum}(x 1[i], i=1 \ldots N): Y:=\operatorname{sum}(y 1[i], i=1 \ldots N)$ : $X X:=\operatorname{sum}(x 2[i], i=1 \ldots N): Y Y:=\operatorname{sum}(y 2[i], i=1 \ldots N): \operatorname{Erx}:=x 1[N]:$ Ery : =y1 $[N]: M:=50: p[1]:=p l o t(X, t=0 . M$, colour=blue, thickness $=$ 3$, numpoint $s=200) ; \mathrm{p}[2]:=\operatorname{plot}(\mathrm{Y}, \mathrm{t}=0 . \mathrm{M}, \mathrm{colour}=\mathrm{blue}$, thickness $=$ 3$, numpoint $s=200) ; \mathrm{p}[3]:=\mathrm{plot}([\mathrm{X}, \mathrm{Y}, \mathrm{t}=0 . \mathrm{M}], \mathrm{colour}=\mathrm{blue}$, thickness $=$ 3 , numpoints=200); $\mathrm{p}[4]:=\mathrm{plot}(\mathrm{XX}, \mathrm{t}=0 . \mathrm{M}, \mathrm{colour}=\mathrm{red}$, thickness $=$ 3 , numpoints $=200) ; \mathrm{p}[5]:=\mathrm{plot}(\mathrm{YY}, \mathrm{t}=0 . \mathrm{M}, \mathrm{colour}=\mathrm{red}$, thickness $=$ 3 , numpoint $\mathrm{s}=200) ; \mathrm{p}[6]:=\mathrm{plot}([\mathrm{XX}, \mathrm{YY}, \mathrm{t}=0 . \mathrm{M}]$, colour=red, thickness $=$ 
3, numpoints=200); $\operatorname{display}(\{\mathrm{p}[1], \mathrm{p}[4]\}) ; \operatorname{display}(\{\mathrm{p}[2], \mathrm{p}[5]\})$;

display ( $\{\mathrm{p}[3], \mathrm{p}[6]\})$;

\section{Appendix 3}

\section{Maple code for plotting Figures 2 and 3:}

restart:with (plots) $: \mathrm{N}:=20:$ \#alpha: $=0.8$ :

$\mathrm{x} 1[0]:=0.4+0.1 * \mathrm{alpha}: \mathrm{dx} 1[0]:=-0.1+0.1 * \mathrm{alpha}:$

$\mathrm{x} 2[0]:=0.6-0.1 * a 1 \mathrm{pha}: \mathrm{dx} 2[0]:=0.1-0.1 * \mathrm{alpha}:$

$\mathrm{y} 1[0]:=0.4+0.1 * \mathrm{alpha}: \mathrm{dy} 1[0]:=-0.1+0.1 * a 1 \mathrm{pha}:$

y2 $[0]:=0.6-0.1 * a l p h a:$ dy2 $[0]:=0.1-0.1 * a l p h a: h:=1.0: k:=0.0: F[1]:=1:$

$\mathrm{a}[\mathrm{x}]:=0: \mathrm{a}[\mathrm{y}]:=0: \mathrm{q}[\mathrm{x}]:=0.4: \mathrm{q}[\mathrm{y}]:=0.4: \mathrm{x} 1[1]:=\mathrm{x} 1[0]:$

$\mathrm{x} 1[2]:=\operatorname{int}(\mathrm{dx} 1[0], \mathrm{t}=0 \ldots t)+\mathrm{h} * \operatorname{int}(\operatorname{int}((\mathrm{a}[\mathrm{x}]-2 * \mathrm{q}[\mathrm{x}] * \cos (2 * \mathrm{t}) /$

$(1-k * \cos (4 * t))) *(x 1[1]), t=0 \ldots t), t=0 \ldots t): x 2[1]:=x 2[0]:$

$x 2[2]:=\operatorname{int}(d x 2[0], t=0 \ldots t)+h * \operatorname{int}(\operatorname{int}((a[x]-2 * q[x] * \cos (2 * t) /$

$(1-\mathrm{k} * \cos (4 * t))) *(\mathrm{x} 2[1]), \mathrm{t}=0 \ldots \mathrm{t}), \mathrm{t}=0 \ldots \mathrm{t}): \mathrm{y} 1[1]:=\mathrm{y} 1[0]:$

$y 1[2]:=\operatorname{int}(\operatorname{dy} 1[0], t=0 \ldots t)-\operatorname{int}(\operatorname{int}((a[y]-2 * q[y] * \cos (2 * t) /$

$(1-\mathrm{k} * \cos (4 * t))) *(\mathrm{y} 1[1]), \mathrm{t}=0 \ldots t), \mathrm{t}=0 \ldots t): \mathrm{y} 2[1]:=\mathrm{y} 2[0]:$

y2 [2] : =int (dy2 [0], t=0 . t ) -int (int ( (a [y] $-2 * q[y] * \cos (2 * t) /$

$(1-k * \cos (4 * t))) *(y 2[1]), t=0 \ldots t), t=0 \ldots t)$ : for $m$ from 3 by 1 to $N$ do

$\mathrm{x} 1[\mathrm{~m}]:=\mathrm{h}^{\wedge}(\mathrm{m}-1) * \operatorname{int}(\operatorname{int}((\mathrm{a}[\mathrm{x}]-2 * \mathrm{q}[\mathrm{x}] * \cos (2 * \mathrm{t}) /$

$(1-\mathrm{k} * \cos (4 * t))) *(\mathrm{x} 1[\mathrm{~m}-1]), \mathrm{t}=0 \ldots \mathrm{t}), \mathrm{t}=0 \ldots \mathrm{t}):$

$\mathrm{x} 2[\mathrm{~m}]:=\mathrm{h}^{\wedge}(\mathrm{m}-1) * \operatorname{int}(\operatorname{int}((\mathrm{a}[\mathrm{x}]-2 * \mathrm{q}[\mathrm{x}] * \cos (2 * \mathrm{t}) /$

$(1-\mathrm{k} * \cos (4 * t))) *(\mathrm{x} 2[\mathrm{~m}-1]), \mathrm{t}=0 \ldots \mathrm{t}), \mathrm{t}=0 \ldots \mathrm{t}):$

$\mathrm{y} 1[\mathrm{~m}]:=-\operatorname{int}(\operatorname{int}((\mathrm{a}[\mathrm{y}]-2 * \mathrm{q}[\mathrm{y}] * \cos (2 * \mathrm{t}) /(1-\mathrm{k} * \cos (4 * t))) *(\mathrm{y} 1[\mathrm{~m}-1])$

$, t=0 \ldots t), t=0 \ldots t):$

y2 $[\mathrm{m}]:=-\operatorname{int}(\operatorname{int}((\mathrm{a}[\mathrm{y}]-2 * \mathrm{q}[\mathrm{y}] * \cos (2 * t) /(1-\mathrm{k} * \cos (4 * t))) *(\mathrm{y} 2[\mathrm{~m}-1])$

$, t=0 . t), t=0 . t):$

end do: $X:=\operatorname{sum}(x 1[i], i=1 \ldots N): Y:=\operatorname{sum}(y 1[i], i=1 \ldots N)$ :

$X X:=\operatorname{sum}(x 2[i], i=1 \ldots N): Y Y:=\operatorname{sum}(y 2[i], i=1 \ldots N): \operatorname{Erx}:=x 1[N]:$

Ery: =y1 $[\mathrm{N}]:$ with (ImageTools): with (plottools):

sX: $=\operatorname{evalf}(\operatorname{subs}(t=3, X))$; sy: =evalf $(\operatorname{subs}(t=3, Y))$;

$\operatorname{sxx}:=\operatorname{evalf}(\operatorname{subs}(t=3, X X)) ; \operatorname{syy}:=\operatorname{evalf}(\operatorname{subs}(t=3, Y Y))$;

$\mathrm{p}[1]:=\mathrm{plot}(\{\mathrm{sx}, \mathrm{sxx}\}, \mathrm{alpha}=0 \ldots 1, \mathrm{color}=\mathrm{blue}$,

numpoints $=200$, linestyle=dashdot, axes $=$ box, axesfont $=$ [TIMES,

16], labelfont $=$ [TIMES, BOLD, 14], thickness $=2)$;

$\mathrm{p}[2]:=\operatorname{plot}(\{\mathrm{sy}, \mathrm{syy}\}, \mathrm{alpha}=0.1, \mathrm{color}=\mathrm{red}$

, numpoint $\mathrm{s}=200,1$ inestyle=dash

, axes $=$ box, labelfont $=[$ SYMBOL $]$, axesfont $=[$ TIMES, 16], labelfont

$=[$ TIMES, BOLD, 14], thickness=2); $\mathrm{q}[1]:=\operatorname{rotate}(\mathrm{p}[1], \mathrm{Pi} / 2):$

$\mathrm{q}[2]:=\operatorname{rotate}(\mathrm{p}[2], \mathrm{Pi} / 2): \operatorname{display}(\mathrm{q}[1], \mathrm{q}[2])$;

plot3d $(\{x, x x\}, a l p h a=0.1, t=0.5$, axes $=$ box, axesfont $=[$ TIMES,

14], labelfont $=[$ TIMES, BOLD, 12] $) ; \operatorname{plot} 3 d(\{Y, Y Y\}, a l p h a=0 \ldots 1$,

$\mathrm{t}=0.5$, axes = box, axesfont = [TIMES, 14], labelfont =

[TIMES, BOLD, 12]);

Competing interests

The authors declare that they have no competing interests.

\section{Authors' contributions}

All authors read and approved the final manuscript.

\section{Author details}

'Department of Mathematics, Faculty of Science, Universiti Putra Malaysia, Serdang, 43400 UPM, Malaysia. ${ }^{2}$ Plasma

Physics and Nuclear Fusion Research School, Nuclear Science and Technology Research Institute (NSTRI), P.O. Box 
14395-836, Tehran, Iran. ${ }^{3}$ Department of Mathematics, Science and Research Branch, Bushehr Branch, Islamic Azad University, Bushehr, Iran. ${ }^{4}$ Institute of Mathematical Research Universiti Putra Malaysia, Serdang, Selangor Darul Ehsan 43400 UPM, Malaysia.

\section{Acknowledgements}

The authors thank the referees for valuable comments and suggestions which improved the presentation of this manuscript.

\section{Received: 15 April 2012 Accepted: 12 February 2013 Published: 1 March 2013}

\section{References}

1. Paul, W, Steinwedel, H, Naturforch, Z: J. Chem. Educ. A8, 448 (1953)

2. Kashanian, F, Nouri, S, Chaharborj, SS, Rizam, ABM: Int. J. Mass Spectrom. 303, 199 (2011)

3. Kiai, SMS, Chaharborj, SS, Bakar, MRA, Fudziah, I: J. Anal. At. Spectrom. 26, 2247 (2011)

4. Chaharborj, SS, Kiai, SMS, Bakar, MRA, Ziaeian, I, Fudziah, I: Int. J. Mass Spectrom. 309, 63 (2012)

5. Chaharborj, SS, Kiai, SMS: J. Mass Spectrom. 45, 111 (2010)

6. March, RE, Todd, JFJ: Practical Aspects of Ion Trap Mass Spectrometry: Chemical, Environmental, and Biomedical Application. CRC Press, New York (1995)

7. March, RE: J. Mass Spectrom. 32, 263 (1997)

8. March, RE: Int. J. Mass Spectrom. 200, 285 (2000)

9. Philip, EM, Denton, MB: J. Chem. Educ. 63, 617 (1986)

10. McLachlan, NW: Theory and Application of Mathieu Functions. Environmental, and Biomedical Application. Clarendon, Oxford (1947)

11. Abbasbandy, S, Allahviranloo, T, Darabi, P: Math. Comput. Appl. 16, 935-946 (2011)

12. Ghanaie, ZA, Moghadam, MM: J. Math. Comput. Sci. 2, 208-221 (2011)

13. Allahviranloo, T, Ahmady, E, Ahmady, N: Int. J. Comput. Math. 86, 730-742 (2009)

14. Allahviranloo, T, Ahmady, E, Ahmady, N: Inf. Sci. 178, 1309-1324 (2008)

15. Barnabas, B, Imre, JR, Attila, LB: Inf. Sci. 177, 1648-1662 (2007)

16. Diamond, P, Kloeden, P: Metric Spaces of Fuzzy Sets: Theory and Applications. World Scientific, Singapore (1994)

17. James, JB, Thomas, F: Fuzzy Sets Syst. 121, 247-255 (2001)

18. Georgiou, DN, Juan, JN, Rosana, RL: Nonlinear Anal., Theory Methods Appl. 63, 587-600 (2005)

19. Marek, TM: Nonlinear Anal., Real World Appl. 13, 860-881 (2012)

20. Khastan, A, Nieto, JJ, Rosana, RL: Fuzzy Sets Syst. 177, 20-33 (2011)

doi:10.1186/1687-1847-2013-45

Cite this article as: Seddighi Chaharborj et al.: Quadrupole mass filter with fuzzy initial conditions. Advances in Difference Equations 2013 2013:45.

\section{Submit your manuscript to a SpringerOpen ${ }^{\circ}$ journal and benefit from:}

- Convenient online submission

Rigorous peer review

- Immediate publication on acceptance

Open access: articles freely available online

- High visibility within the field

- Retaining the copyright to your article 